\title{
ON LINEARIZATION OF LIE GROUP EXTENSIONS
}

\section{G. HOCHSCHILD}

1. Introduction. If $S$ and $G$ are given connected Lie groups it is natural to attempt the classification of the Lie group extensions of $S$ by $G$ ( $S$ being the kernel and $G$ the image) by considering the associated Lie algebra extensions of the Lie algebra $\subseteq$ of $S$ by the Lie algebra (5) of $G$. However, even in the case where $S$ is a vector group, it is well known that the natural map from the set of equivalence classes of Lie group extensions of $S$ by $G$ to the set of equivalence classes of Lie algebra extensions of $\subseteq$ by $(5)$ is generally neither injective nor surjective. A remedy for this is found in the cohomology theory of Lie groups.

Let $P$ be a maximal compact subgroup of $G$, and let $\mathfrak{B}$ be its Lie algebra. It was shown by van Est [5] that the cohomology of $G$ in the vector group $S$ (based on differentiable cochains) is isomorphic with the relative cohomology for $(\mathfrak{B}, \mathfrak{B})$ in $\mathfrak{S}$. This result, for the 2dimensional cohomology groups, can be interpreted to show that the group of equivalence classes or extensions of $S$ by $G$ is isomorphic with the group of equivalence classes of (relative) (S, $\mathfrak{P}$ )-extensions of $\mathfrak{S}$, as defined below.

Actually, we shall not borrow any results from the cohomology theory, but we shall proceed directly within the framework of extensions to establish the following more general result. Let $G$ and $S$ be connected Lie groups, let $P$ be a maximal compact subgroup of $G$, and assume that $S$ is solvable and simply connected. We shall show that a Lie group extension of $S$ by $G$ determines in the natural fashion not only a Lie algebra extension of $\mathbb{S}$ by $\mathbb{S}$ but the more detailed structure of an equivalence class of $(\mathfrak{B}, \mathfrak{B})$-extensions of $\mathfrak{S}$, and that this yields a one to one correspondence between the equivalence classes of extensions of $S$ by $G$ and the equivalence classes of those $(\mathfrak{B}, \mathfrak{B})$-extensions of $\mathfrak{\subseteq}$ which are compatible with continuous representations of $P$ by Lie algebra automorphisms of $\mathfrak{S}$.

As in the cohomology theory of Lie groups, the key to this result is the theory of the maximal compact subgroups of Lie groups; in particular, the theorem that if $G$ is a Lie group with only a finite number of connected components then $G$ has a compact subgroup $P$ and closed 1-dimensional vector subgroups $H_{1}, \cdots, H_{k}$ such that the $\operatorname{map}\left(p, h_{1}, \cdots, h_{k}\right) \rightarrow p h_{1} \cdots h_{k}$ is an analytic manifold isomorphism of the Cartesian product $P \times H_{1} \times \cdots \times H_{k}$ onto $G$ (see $[2 ; 3]$ ).

Received by the editors November 2, 1961. 
If $S$ is a vector group, the main result can be extended to the case where the assumption that $G$ be connected is relaxed to the assumption that $G$ have only a finite number of connected components; one must merely replace the set of equivalence classes of $(\mathcal{B}, \mathfrak{B})$-extensions of $\subseteq$ by the subset of those classes that are fixed under the natural action of $P / P_{1}$, where $P_{1}$ is the connected component of the identity in $P$. In the case of a noncommutative kernel $S$, this extension appears to lead to difficulties of formulation and proof. In any case, it seems reasonable here, in the interest of simplicity, to confine one's attention to the case of connected Lie groups.

2. Position of maximal compact subgroups. Our first result may be viewed as a refinement of the well-known fact that extensions of simply connected solvable groups by compact groups are trivial (see [1, Lemma 8.1]). It provides the additional structure needed for the main result.

TheOREM 1. Let $S$ be a connecied, simply connected and solvable Lie group, and let $G$ be a Lie group whose group of components $G / G_{1}$ is finite. Let $P$ be a maximal compact subgroup of $G$. Let $(E, \phi)$ be a Lie group extension of $S$ by $G$, i.e., $\phi$ is a continuous, and hence analytic, epimorphism of the Lie group $E$ onto $G$, and the kernel of $\phi$ coincides with $S$. Then there is an analytic map $\rho: G \rightarrow E$ such that $\phi \circ \rho$ is the identity map on $G$ and, for every $g \in G$ and every $p \in P, \rho(g p)=\rho(g) \rho(p)$ and $\rho(p g)=\rho(p) \rho(g)$. Given any two continuous monomorphisms $\gamma$ and $\gamma^{\prime}$ of $P$ into $E$ such that $\phi \circ \gamma$ and $\phi \circ \gamma^{\prime}$ coincide with the identity map on $P$, there is an element $s$ in $S$ such that $\gamma^{\prime}(p)=s \gamma(p) s^{-1}$, for every $p \in P$.

Proof. There are closed 1-dimensional vector subgroups $H_{1}, \cdots$, $H_{k}$ of $G$ such that the $\operatorname{map}\left(p, h_{1}, \cdots, h_{k}\right) \rightarrow p h_{1} \cdots h_{k}$ is an analytic manifold isomorphism of $P \times H_{1} \times \cdots \times H_{k}$ onto $G$. Using that every extension of $S$ by $P$ or by an $H_{i}$ is trivial, we see that there is an analytic map $\rho_{0}: G \rightarrow E$ such that $\phi \circ \rho_{0}$ is the identity map on $G$ and $\rho_{0}(p g)=\rho_{0}(p) \rho_{0}(g)$, whenever $p \in P$.

Let $S_{0}=S$ and define $S_{1}, S_{2}, \cdots$ by making $S_{i+1}$ the commutator subgroup of $S_{i}$, for each $i$. Suppose that we have already found an analytic map $\rho_{n}: G \rightarrow E$ such that $\phi \circ \rho_{n}$ is the identity map on $G$, and $\rho_{n}(p g)=\rho_{n}(p) \rho_{n}(g)$ and $\rho_{n}(g)^{-1} \rho_{n}(g p) \rho_{n}(p)^{-1} \in S_{n}$, whenever $p \in P$. Let $\sigma(p)(g)$ denote the coset mod. $S_{n+1}$ of $\rho_{n}(g)^{-1} \rho_{n}(g p) \rho_{n}(p)^{-1}$. Then the map $(p, g) \rightarrow \sigma(p)(g)$ is an analytic map of $P \times G$ into the vector group $S_{n} / S_{n+1}$. Moreover, $\sigma(p)(g)=\sigma(p)\left(p_{1} g\right)$, whenever $p_{1} \in P$. We regard $S_{n} / S_{n+1}$ as a representation space for $P$ such that, if $p \in P$, the automorphism of $S_{n} / S_{n+1}$ corresponding to $p$ is induced by the automor- 
phism $s \rightarrow \rho_{n}(p) s \rho_{n}(p)^{-1}$ of $S$. Writing this $u \rightarrow p \cdot u$, we verify directly that, for all $p_{1}$ and $p_{2}$ in $P$ and all $g$ in $G$,

$$
p_{1} \cdot\left[\sigma\left(p_{2}\right)\left(g \dot{p}_{1}\right)\right]-\sigma\left(p_{1} p_{2}\right)(g)+\sigma\left(p_{1}\right)(g)=0 .
$$

Let $I_{P}$ denote the normalized Haar integral for the continuous maps of $P$ into the vector space $S_{n} / S_{n+1}$, and define the map $\tau: G$ $\rightarrow S_{n} / S_{n+1}$ by $\tau(g)=I_{P}\left(\sigma_{g}\right)$, where $\sigma_{g}(p)=\sigma(p)(g)$. Then $\tau$ is evidently an analytic map, and $\tau(p g)=\tau(g)$, whenever $p \in P$. Moreover, our above identity for $\sigma$ gives

$$
p \cdot \tau(g p)-\tau(g)+\sigma(p)(g)=0 .
$$

Since $S$ is simply connected, there is an analytic map $\psi: S_{n} / S_{n+1}$ $\rightarrow S_{n}$ such that $\psi(u) S_{n+1}=u$, for every $u \in S_{n} / S_{n+1}$, and $\psi(0)=1$. Let $\zeta=\psi \circ \tau$ and define $\rho_{n+1}(g)=\rho_{n}(g) \zeta(g)$. Note that $\sigma_{p}=0$ if $p \in P$. Hence $\tau$ vanishes identically on $P$, so that $\zeta$ maps $P$ onto the identity element of $S_{n}$. Thus $\rho_{n+1}$ coincides with $\rho_{n}$ on $P$.

Now let $p \in P$ and $g \in G$. Then we find that

$$
\rho_{n+1}(p g)=\rho_{n}(p g) \zeta(p g)=\rho_{n}(p) \rho_{n}(g) \zeta(g)=\rho_{n+1}(p) \rho_{n+1}(g),
$$

and

$$
\begin{aligned}
\rho_{n+1}(g)^{-1} \rho_{n+1}(g p) \rho_{n+1}(p)^{-1} & =\zeta(g)^{-1} \rho_{n}(g)^{-1} \rho_{n}(g p) \zeta(g p) \rho_{n}(p)^{-1} \\
& =\zeta(g)^{-1}\left[\rho_{n}(g)^{-1} \rho_{n}(g p) \rho_{n}(p)^{-1}\right] \rho_{n}(p) \zeta(g p) \rho_{n}(p)^{-1} .
\end{aligned}
$$

The coset $\bmod S_{n+1}$ of the last expression is equal to

$$
-\tau(g)+\sigma(p)(g)+p \cdot \tau(g p)=0 .
$$

Thus we have $\rho_{n+1}(g)^{-1} \rho_{n+1}(g p) \rho_{n+1}(p)^{-1} \in S_{n+1}$. Since $S$ is solvable, there is an index $m$ such that $S_{m}=(1)$, and then $\rho_{m}$ evidently satisfies the requirements of the first part of Theorem 1 .

Now we prove the second part by a similar approximation procedure. Suppose that we have already found an element $s_{n} \in S$ such that $\gamma^{\prime}(p)=\delta_{n}(p) s_{n} \gamma(p) s_{n}^{-1}$, with some $\delta_{n}(p) \in S_{n}$, for every $p \in P$. For $n=0$, this is indeed the case, with $s_{0}=1$. Let $\beta(p)$ denote the coset $\bmod S_{n+1}$ of $\delta_{n}(p)$. Then $\beta$ is evidently a continuous map of $P$ into the vector group $S_{n} / S_{n+1}$. We regard $S_{n} / S_{n+1}$ as a representation space for $P$ such that, if $p \in P$, the corresponding automorphism of $S_{n} / S_{n+1}$ is induced by the automorphism $s \rightarrow \gamma^{\prime}(p) s \gamma^{\prime}(p)^{-1}$ of $S_{n}$. Now one verifies directly that $p_{1} \cdot \beta\left(p_{2}\right)-\beta\left(p_{1} p_{2}\right)+\beta\left(p_{1}\right)=0$, for all $p_{1}$ and $p_{2}$ in $P$. Hence we obtain $p \cdot u-u+\beta(p)=0$, for every $p \in P$, where $u=I_{P}(\beta)$. Let $s$ be a representative in $S_{n}$ for $u$. Then, for every $p \in P$, we may write

$$
\delta_{n}(p)=\delta_{n+1}(p) s s_{n} \gamma(p) s_{n}^{-1} s^{-1} s_{n} \gamma(p)^{-1} s_{n}^{-1}, \text { with } \delta_{n+1}(p) \in S_{n+1} .
$$


Hence we have

$$
\gamma^{\prime}(p)=\delta_{n+1}(p) s_{n+1} \gamma(p) s_{n+1}^{-1}, \quad \text { where } s_{n+1}=s s_{n} \in S .
$$

This evidently suffices to establish the second part of Theorem 1.

In particular, it follows from Theorem 1 that a Lie group extension of $S$ by $G$ determines a unique equivalence class of analytic representations of $P$ by analytic automorphisms of $S$, where we say that two such representations $\alpha$ and $\alpha^{\prime}$ are equivalent if there is an inner automorphism $\zeta$ of $S$ such that $\alpha(p)=\zeta \circ \alpha^{\prime}(p) \circ \zeta^{-1}$, for every $p \in P$. In fact, these representations $\alpha$ are obtained from the monomorphisms $\gamma$ of the second part of Theorem $1 ; \alpha(p)(s)=\gamma(p) s \gamma(p)^{-1}$, for every $p$ in $P$ and every $s$ in $S$. We shall refer to this structure by saying that $S$ is a representation group for $P$. Since $S$ is simply connected, it is equivalent to say that the Lie algebra $\subseteq$ of $S$ is a representation Lie algebra for $P$. Evidently, these structures depend only on the equivalence class of the given Lie group extension of $S$ by $G$.

For the later construction of a Lie group extension from a given Lie algebra extension, we shall require the following lemma.

Lemma. Let $G$ be a connected Lie group, and let $P$ be a maximal compact subgroup of $G$. Let $\delta: G^{*} \rightarrow G$ be the universal covering epimorphism for $G$. Then $\delta^{-1}(P)$ is connected and simply connected.

Proof. Let $\psi: P^{*} \rightarrow P$ be the universal covering epimorphism for $P$. $G$ contains a closed, connected and simply connected subspace $U$ such that the map $(u, p) \rightarrow u p$ of $U \times P$ into $G$ is a homeomorphism and $U$ contains the identity element of $G$. Consider the map $\psi^{+}: U \times P^{*}$ $\rightarrow G$ given by $\psi^{+}((u, x))=u \psi(x)$, for every $u \in U$ and every $x \in P^{*}$. This is evidently a covering of $G$ by the simply connected space $U \times P^{*}$. Hence there exists a homeomorphism $h$ of $U \times P^{*}$ onto $G^{*}$ such that $h((1,1))=1$ and $\delta \circ h=\psi^{+}$. Clearly, $h\left(1 \times P^{*}\right) \subset \delta^{-1}(P)$. Conversely, suppose that $u \in U, x \in P^{*}$ and $\delta(h((u, x))) \in P$. Then $\psi^{+}((u, x)) \in P$, i.e., $u \psi(x) \in P$, whence $u=1$. Thus $h\left(1 \times P^{*}\right)=\delta^{-1}(P)$. This shows that $\delta^{-1}(P)$ is homeomorphic with $P^{*}$ and thus that it connected and simply connected.

3. Relative Lie algebra extensions. Let $\mathfrak{S}, \mathfrak{S}, \mathfrak{B}$ be real Lie algebras, with $\mathfrak{B} \subset(\mathfrak{S}$. A $(\mathfrak{S}, \mathfrak{B})$-extension of $\mathfrak{S}$ is a triple $(\mathbb{F}, \sigma, \tau)$, where $\mathbb{E}$ is a Lie algebra containing $\mathfrak{S}, \sigma$ is a Lie algebra epimorphism of $\mathbb{E}$ onto $\mathfrak{B}$ with kernel $\mathfrak{S}$, and $\tau$ is a Lie algebra monomorphism of $\mathfrak{B}$ into (5 that is extendible to a linear map $\rho: B \rightarrow(F)$ such that $\sigma \circ \rho$ is the identity map on $B$ and $\rho([x, y])=[\rho(x), \rho(y)]$ whenever $x \in \mathfrak{B}$. Let $A(\xi)$ denote the group of all Lie algebra automorphisms of $\leftleftarrows$, and let $A \Subset(\xi)$ denote the analytic subgroup of $A(\xi)$ whose Lie algebra 
consists of the derivations $D_{x}$, with $x \in \Im$, where $D_{x}(y)=[x, y]$, for every $y \in \mathbb{F}$. We say that two $(\mathfrak{F}, \mathfrak{B})$-extensions $(\&, \sigma, \tau)$ and $\left(\mathcal{F}^{\prime}, \sigma^{\prime}, \tau^{\prime}\right)$ of $\subseteq$ are equivalent if there is a Lie algebra isomorphism $\alpha$ of $\mathbb{E}$ onto $\xi^{\prime}$ leaving the elements of $\Im$ fixed and such that $\sigma^{\prime} \circ \alpha=\sigma$ and $\alpha \circ \tau=\gamma \circ \tau^{\prime}$, with some $\gamma \in A \Subset\left(\xi^{\prime}\right)$.

Now suppose that $\mathfrak{S}, \mathfrak{B}, \mathfrak{B}$ are the Lie algebras of connected Lie groups $S, G, P$, respectively, where $S$ is simply connected and solvable and where $P$ is a maximal compact subgroup of $G$. A (B), $\mathfrak{B})$-extension $(\mathfrak{F}, \sigma, \tau)$ of $\mathfrak{S}$ determines a Lie algebra homomorphism of $\mathfrak{B}$ into the derivation algebra $D(\Im)$ of $\Im$ sending an element $x$ of $\mathfrak{P}$ into the derivation $y \rightarrow[\tau(x), y]$ of $\widetilde{S}$. If $S$ is equipped with the structure of a representation group for $P$ then $\subseteq$ inherits the structure of a representation Lie algebra for $P$. We say that $(\mathcal{E}, \sigma, \tau)$ is compatible with this structure if the Lie algebra homomorphism $\mathfrak{B} \rightarrow D(\Im)$ that is determined by $\tau$ is the differential of a homomorphism $P \rightarrow A$ (ऽ) belonging to the given structure of $\subseteq$ as a representation Lie algebra for $P$. Clearly, this compatibility depends only on the equivalence class of $(\xi, \sigma, \tau)$. If $(E, \phi)$ is a Lie group extension of $S$ by $G, \sigma$ is the differential of $\phi$ and $\tau$ is the differential of a monomorphism $P \rightarrow E$ inverse to $\phi$, then it follows immediately from 'Theorem 1 that $(\&, \sigma, \tau)$ is a $(\mathfrak{S}, \mathfrak{B})$-extension of $\subseteq$ and is compatible with the structure of $\mathfrak{S}$ as a representation Lie algebra for $P$ that is determined by $(E, \phi)$. Moreover, the second part of Theorem 1 shows that the equivalence class of $(\&, \sigma, \tau)$ is determined uniquely by $(E, \phi)$ and depends only on the equivalence class of $(E, \phi)$.

Thus we have a map, called the canonical map, from the set of equivalence classes of Lie group extensions of $S$ by $G$ to the set of equivalence classes of $(\mathfrak{S}, \mathfrak{B})$-extensions of $\subseteq$ compatible with some structure of $\mathfrak{S}$ as a representation Lie algebra for $P$.

4. The inverse of the canonical map. Let $(\mathfrak{F}, \sigma, \tau)$ be a $(\mathfrak{S}, \mathfrak{P})$ extension of $\subseteq$ that is compatible with some structure of $\subseteq$ as a representation Lie algebra for $P$. Let $E^{*}$ be the simply connected Lie group with Lie algebra $\left(\xi\right.$, and let $\delta: G^{*} \rightarrow G$ be the universal covering epimorphism for $G$. Let $\sigma^{*}: E^{*} \rightarrow G^{*}$ be the analytic epimorphism whose differential is the given Lie algebra epimorphism $\sigma:(\& \rightarrow$ (s). Since $G^{*}$ is simply connected, the kernel of $\sigma^{*}$ is connected and thus coincides with the analytic subgroup $S$ of $E^{*}$ whose Lie algebra is $\subseteq$. By the lemma of $\$ 1$, the universal covering group $P^{*}$ of $P$ may be identified with the closed analytic subgroup $\delta^{-1}(P)$ of $G^{*}$ whose Lie algebra is $\mathfrak{P}$. Let $\tau^{*}: P^{*} \rightarrow E^{*}$ be the analytic monomorphism whose differential is the Lie algebra monomorphism $\tau: \mathfrak{\beta \rightarrow}$. Since $\sigma \circ \tau$ is 
the identity map on $\mathfrak{B}, \sigma^{*} \circ \tau^{*}$ is the identity map on $P^{*}$. Since $S$ is homeomorphic with Euclidean space, it follows from a standard result on cross-sections of fibre bundles ([4, Theorem 12.2]) that $\tau^{*}$ can be extended to a continuous map $\rho^{*}: G^{*} \rightarrow E^{*}$ such that $\sigma^{*} \circ \rho^{*}$ is the identity map on $G^{*}$. Hence $E^{*}$, as a topological space, is a Cartesian product $S \times U$, where $1 \times U$ contains $\tau^{*}\left(P^{*}\right)$ and is mapped homeomorphically onto $G^{*}$ by $\sigma^{*}$. It follows that $\tau^{*}$ maps $P^{*}$ homeomorphically onto the subspace $\tau^{*}\left(P^{*}\right)$ of $E^{*}$.

Let $F$ denote the kernel of the covering epimorphism $\delta: G^{*} \rightarrow G$. We know from the lemma of $\S 1$ that $F \subset P^{*}$ and is the fundamental group of $P$ (as well as that of $G$ ). In particular, $F$ is a discrete subgroup of $P^{*}$. Hence $\tau^{*}(F)$ is a discrete subgroup of $E^{*}$.

By assumption, $\tau$ has an extension $\rho:$ (s) $\rightarrow$ ( $)$ such that $\sigma \circ \rho$ is the identity map on $\mathbb{B}$ and $\rho([x, y])=[\tau(x), \rho(y)]$, whenever $x \in \mathfrak{B}$. Let $u \rightarrow u^{*}$ denote the adjoint representation of a Lie group on its Lie algebra. Let $x \in \mathfrak{P}, y \in \mathcal{S}$. Then we have, if $D_{v}$ denotes the derivation effected by $v$,

$$
\begin{aligned}
\tau^{*}\left(\exp _{P^{*}}(x)\right)^{*}(\rho(y)) & =\exp _{E^{*}}(\tau(x))^{*}(\rho(y))=\operatorname{Exp}\left(D_{\tau(x)}\right)(\rho(y)) \\
& =\rho\left(\operatorname{Exp}\left(D_{x}\right)(y)\right)=\rho\left(\exp _{G^{*}}(x)^{*}(y)\right) .
\end{aligned}
$$

Hence, for every $u \in P^{*}, \tau^{*}(u)^{*}(\rho(y))=\rho\left(\delta(u)^{*}(y)\right)$. If $u \in F$ this gives $\tau^{*}(u)^{*}(\rho(y))=\rho(y)$, for every $y \in(s$.

On the other hand, since the homomorphism of $\mathfrak{B}$ into $D(\mathfrak{S})$ obtained from $\tau$ is the differential of a homomorphism of $P$ into $A(\mathfrak{S})$, the restriction of $\tau^{*}(u)^{*}$ to $\mathfrak{S}$ is the identity automorphism of $\mathfrak{S}$, whenever $u \in F$. Hence we conclude, since $\mathbb{E}=\Im+\rho(\$)$, that $\tau^{*}(u)^{*}$ is the identity automorphism of $\mathbb{E}$, whenever $u \in F$. This means that $\tau^{*}(F)$ lies in the center of $E^{*}$.

Now let $E=E^{*} / \tau^{*}(F)$. The epimorphism $\sigma^{*}: E^{*} \rightarrow G^{*}$ induces an epimorphism $\phi: E \rightarrow G^{*} / F=G$, and the kernel of $\phi$ is the canonical image of $S$ in $E$, which may still be identified with $S$, because $S \cap \tau^{*}\left(P^{*}\right)=(1)$. Thus we have a Lie group extension $(E, \phi)$ of $S$ by $G$, and it is clear from its construction that $(\&, \sigma, \tau)$ belongs to the equivalence class of $(\mathfrak{B}, \mathfrak{P})$-extensions of $\mathfrak{S}$ determined by $(E, \phi)$. We have shown, therefore, that the canonical map from Lie group extensions to relative Lie algebra extensions is subjective.

Now let $(E, \phi)$ and $\left(E^{\prime}, \phi^{\prime}\right)$ be two Lie group extensions of $S$ by $G$.

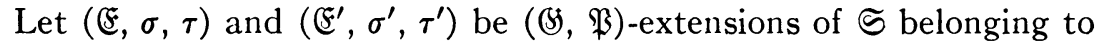
the equivalence classes determined by $(E, \phi)$ and $\left(E^{\prime}, \phi^{\prime}\right)$, respectively. Suppose that these are equivalent, say by an isomorphism $\alpha: \mathbb{E} \rightarrow \mathbb{F}^{\prime}$. Let $E^{*}$ and $E^{* *}$ be the universal covering groups of $E$ and 
$E^{\prime}$, respectively, and let $\alpha^{*}$ be the analytic isomorphism of $E^{*}$ onto $E^{\prime *}$ whose differential is $\alpha$. If we identify $S$ with the analytic subgroups of $E^{*}$ and $E^{\prime *}$ whose Lie algebras are $\mathfrak{S}$, it is clear that $\alpha^{*}$ leaves the elements of $S$ fixed.

We have $\alpha \circ \tau=\gamma \circ \tau^{\prime}$, where $\gamma \in A \Subset\left(\mathbb{E}^{\prime}\right)$. Hence $\alpha^{*} \circ \tau^{*}=\gamma^{*} \circ \tau^{\prime *}$, where $\gamma^{*}$ is the inner automorphism of $E^{\prime *}$ effected by an element of $S$. We have seen that, if $F$ is the fundamental group of $P$, then $\tau^{\prime *}(F)$ is the fundamental group of $E^{\prime}$ and, in particular, lies in the center of $E^{\prime *}$. Hence we see that $\alpha^{*}$ sends the fundamental group $\tau^{*}(F)$ of $E$ onto the fundamental group $\tau^{\prime *}(F)$ of $E^{\prime}$. It follows that $\alpha^{*}$ induces an isomorphism of $E$ onto $E^{\prime}$, which is evidently an equivalence isomorphism of the extension $(E, \phi)$ onto the extension $\left(E^{\prime}, \phi^{\prime}\right)$. Thus we have proved the following result.

Theorem 2. Let $G, S$ and $P$ be connected Lie groups, with $P$ a maximal compact subgroup of $G$, and $S$ simply connected and solvable. Then the canonical map sends the set of equivalence classes of Lie group extensions of $S$ by $G$ in a one to one fashion onto the set of equivalence classes of those $(\mathfrak{B}, \mathfrak{B})$-extensions of $\mathbb{S}$ which are compatible with some structure of $\mathfrak{S}$ as a representation Lie algebra for $P$.

\section{REFERENCES}

1. G. Hochschild and G. D. Mostow, Representations and representative functions of Lie groups, Ann. of Math. 66 (1957), 495-542. 558.

2. K. Iwasawa, On some types of topological groups, Ann. of Math. 50 (1949), 507-

3. G. D. Mostow, Self-adjoint group, Ann. of Math. 62 (1955), 44-55.

4. N. Steenrod, The topology of fibre bundles, Princeton Univ. Press, Princeton, N. J., 1951.

5. W. T. van Est, On the algebraic cohomology concepts in Lie groups. II, Nederl. Akad. Wetensch. Proc. Ser. A, 58 (1955), 286-294.

University of California, Berkeley 